\title{
Coaches' knowledge and awareness of spit tobacco use among youth athletes: results of a 2009 Ontario survey
}

\section{J. H. C. Skinner, MSc (1); S. J. Bobbili, MPH (2)}

This article has been peer reviewed.

Abstract

Introduction: Public health professionals have become concerned that spit tobacco (ST) use among athletes is increasing. However, little is known about the issue in Canada, particularly among youth.

Methods: The Not to Kids Coalition and the Coaches Association of Ontario surveyed coaches regarding ST knowledge and awareness and their perceived roles as coaches in influencing ST use among their athletes. Surveys were distributed electronically to individuals who coached male and female youth aged 9 to 18 years in baseball, basketball, football, soccer, and track and field, in Ontario.

Results: Almost all of the surveyed coaches responded correctly to questions about the health effects of ST use, and about $80 \%$ of respondents answered correctly to the question about legislation associated with ST and youth.

Conclusion: Most coaches are interested in receiving information about ST, particularly the health effects of ST use and how to prevent ST use among athletes. Multiple formats should be used to provide information to coaches, including both electronic and hard copy materials.

Keywords: tobacco, smokeless chewing tobacco, youth, sport, mentor, coach

\section{Introduction}

Anecdotal observations and local survey results shared at Not to Kids Coalition (NTK) meetings between 2006 and 2007 have indicated that the use of oral (spit) tobacco is increasing among sports participants. ${ }^{1}$ The purpose of our research is to assess the knowledge level of Ontario coaches about spit tobacco (ST) products and to assess their perceptions of ST use among the athletes they coach. This study is not intended to measure prevalence; it was undertaken to direct health promotion initiatives that prevent initiation and promote cessation of the use of ST products among youth. The study was completed through an online survey of amateur coaches who work with children and youth in Ontario.

This project directly addresses legislation mandated by the Ontario government: to work with priority populations to adopt tobacco-free living and reduce the burden of preventable chronic diseases. It also addresses the requirement of Boards of Health to monitor emerging trends in tobacco use. $^{2}$

\section{Background}

Spit tobacco: use and risks

Smokeless tobacco is defined as tobacco products that are administered without being burned. ${ }^{3}$ Smokeless tobacco is mainly found in two forms: oral (moist) snuff, which is powdered, and chewing tobacco, which is coarsely cut. ${ }^{4}$ Those who use smokeless tobacco either place a "pinch" of snuff between their gum and their lip or cheek or chew a "wad" of chewing tobacco. The released nicotine is absorbed through membranes in the mouth. ${ }^{4}$ The term spit tobacco (ST) is used here to refer to both these forms of tobacco.

Like all tobacco products, ST is associated with many adverse health outcomes. Tobacco-specific nitrosamines (cancercausing chemicals) have been linked to oral cancers in humans. ${ }^{5}$ Specifically, ST use has been shown to cause leukoplakia, white patches and lesions on the cheeks, gums or tongue that may lead to oral cancer. ${ }^{5}$ ST use can also lead to gum disease and tooth decay and is associated with increased mortality from cardiovascular disease and stroke. ${ }^{6,7}$ Of particular concern is that ST may be more addictive than cigarettes; compared to cigarette smokers, ST users are exposed to large amounts of nicotine over longer periods. ${ }^{5}$

\section{Use of spit tobacco among youth}

The NTK is a network of 31 public health units from across Ontario whose aim is to reduce youth access to and use of tobacco

\section{Author references:}

1. Haliburton, Kawartha, Pine Ridge District Health Unit, Port Hope, Ontario, Canada 2. Centre for Addiction and Mental Health, Toronto, Ontario, Canada

Correspondence: Jennifer Hope Campin Skinner, Haliburton, Kawartha, Pine Ridge District Health Unit, 200 Rose Glen Road, Port Hope, ON L1A 3V6; Tel.: (905) 885-9100; Fax: (905) 885-9551; Email: jskinner@hkpr.on.ca 
products. NTK recently conducted surveys and focus groups among Ontario youth aged 14 to 19 years. These indicated that youth perceive ST as a safer alternative to cigarette smoking because it produces no smoke. ${ }^{1}$ Moreover, youth respondents failed to identify many of the health-related consequences of using ST. ${ }^{1}$

In North America and Europe, ST use is not as prevalent as cigarette smoking, but it may be increasing due to the introduction of smoking bans in public spaces. ${ }^{7}$ According to the 2008 Canadian Tobacco Use Monitoring Survey, $1 \%$ of youth aged 15 to 19 years ( $\mathrm{n}=30000)$ and $1 \%$ of young adults aged 20 to 24 years $(n=27000)$ reported using ST in the preceding 30 days. $^{8}$ However, in the 2008/2009 Youth Smoking Survey, $5 \%$ of Canadian youth in grades 6 through $12(\mathrm{n}=2600)$ and $4 \%$ of Ontario students in grades 6 through 12 $(n=360)$ reported having "ever tried" ST.

In the United States, the National Survey on Drug Use and Health reported that ST use among persons aged 12 or older remained stable at between $3.0 \%$ and $3.3 \%$ from 2002 to 2007. ${ }^{10}$ However, ST use increased among certain subpopulations, specifically adolescent males, from 3.4\% in 2002 to $4.4 \%$ in 2007, and was higher among those residing in rural areas. ${ }^{10}$

Age, gender, geography, ethnicity and education are all factors related to the prevalence of ST use. The 2009 National Youth Risk Behavior Survey estimated the prevalence of ST use among American high school students at $8.9 \% .^{11}$ This prevalence varied across states, ranging from $4.9 \%$ in Hawaii to $16.2 \%$ in Wyoming, and was higher among male compared to female high school students and among White compared to Black and Hispanic students. ${ }^{11}$ The prevalence among White male students was very high, at $20.1 \%$. ${ }^{11}$

Goebel et al. identified several correlates to ST use among fifth, eighth and eleventh graders in the United States, including having a family member not living at home who uses ST, having a friend who uses ST, playing football, trying cigarettes in the past, and having parents that permit ST use at home. ${ }^{12}$

\section{Use of spit tobacco in sport}

To determine how ST use varies across sports, a 2001 National Collegiate Athletic Association (NCAA) study asked male athletes (17-20 years) to report their ST use in the preceding 30 days. ST use was reported as follows: baseball, $41 \%$; wrestling, $39 \%$; ice hockey, $35 \%$; lacrosse, $32 \%$; football, $29 \%$; golf, $27 \%$; water polo, $25 \%$; soccer, $20 \%$; track and field, $17 \%$; tennis, $13 \%$; and basketball, $12 \% .{ }^{13}$

Research indicates that adolescents who participate in organized sports, while less likely to smoke cigarettes, are more likely to use ST. ${ }^{14,15}$ Castrucci et al. reported that adolescents in grades 9 through 12 who participate in organized sports have $33 \%$ increased odds of ever using ST and 76\% increased odds of currently using ST. ${ }^{14}$ Rigotti et al. reported similar findings in their study of 14138 students aged 18 to 24 years at 119 colleges in the United States. ${ }^{16}$ The researchers found that intercollegiate athletes were more likely to use ST than cigarettes and suggested that athletes may be using ST instead of cigarettes as a substitute form of nicotine. ${ }^{16}$

The NCAA conducted a study of 1985 teams through 1032 member institutions that produced 19676 responses; they found the prevalence of ST use to be $16.3 \% .^{17}$ Among athletes who use ST, more than $50 \%$ began in high school; however, approximately $10 \%$ began using ST in junior high school or earlier. ${ }^{17}$

College student-athletes reported using recreational drugs, such as ST, primarily for recreational or social reasons $(46.8 \%)$ or "to feel good" (28.1\%). ${ }^{18}$ Other reasons given included to help deal with the stress of college life and college athletics (21.2\%), to improve athletic performance $(2.0 \%)$ and to fit in with the team $(1.8 \%) .{ }^{17}$

\section{The role of coaches}

Coaches play the role of teacher, mentor, role model, friend and leader in the community. ${ }^{19}$ They have a strong influence over team values and norms and, as role models, can have a marked effect on shaping the habits of children and young adults. ${ }^{20}$
Walsh et al. confirmed coaches' influence on athletes, specifically in regard to ST use. ${ }^{21}$ Their findings indicate that male high school baseball players were three to four times more likely to use ST if they saw their high school coach or father using ST. ${ }^{21}$

Coaches have the ability to encourage uptake as well as to discourage ST use. They are key players in the effort to decrease ST use among athletes because of their access to players at different stages of ST use (e.g. initiation, experimentation, regular use). ${ }^{22}$ Of the coaches surveyed by Horn et al., $80 \%$ agree that they play vital roles in preventing ST use among athletes, while $86 \%$ believe they could assist youth with ST cessation. ${ }^{22}$ Coaches report using several strategies to address ST use among their athletes, including advising athletes to quit, informing athletes about the health hazards of ST use, and informing athletes' parents or school principals. ${ }^{22}$

\section{Methods}

NTK partnered with the Coaches Association of Ontario (CAO), a non-profit coach-led organization that provides development opportunities and educational resources for coaches, to develop and electronically distribute a survey to amateur coaches in June 2009. The purpose of the survey was (1) to assess coaches' general knowledge and awareness of ST use and (2) to better understand coaches' perceptions of their roles in influencing and preventing ST use among their athletes.

All of the coaches surveyed were members of amateur sport organizations. As such, it is assumed that they were unpaid volunteers at all levels of all sports and coached their athletes on their own time.

Members of CAO and NTK and an epidemiologist decided on the criteria for participating in the survey and developed the survey through an iterative process of identifying content and refining questions. Eligibility criteria, including the rationale for inclusion and exclusion of coaches, were as follows:

- Participants were at least 18 years of age. Anyone younger than 18 would have required more extensive consent procedures. 
- Participants coached at least one of the following: hockey, baseball, soccer, basketball, football, or track and field. $\mathrm{CAO}$, the primary vehicle for initial data collection, only included coaches of these sports in their membership.

- Participants coached youth aged between 9 and 18 years. A broad age group was chosen as little research has been conducted regarding ST among youth athletes, and none to date has included youth aged less than 12 years.

The surveys were distributed electronically using SurveyMonkey, an Internet-based survey development tool. Responses were encrypted and housed on the SurveyMonkey web server.

CAO members who met the eligibility criteria were the initial target population. The sample size estimate of 321 was determined based on the CAO membership size and given a margin of error of $5 \%$ and a confidence interval of $95 \%$ (Table 1).

CAO informed their members about the project and supplied the survey link in their monthly e-newsletter on June 11, 2009. Respondents were offered the opportunity to win one of four $\$ 50$ gift certificates or various anti-tobacco promotional items as an incentive to participate, the winners being selected by a random draw following the survey end date.

Two weeks after the initial e-newsletter distribution by CAO, there were 79 completed surveys. A reminder about the survey was included in the July e-newsletter to CAO members. Following this communication, the survey completion rate remained poor. As a result, the survey link was also distributed to the provincial governing bodies of the targeted sports: Ontario Baseball Association, Ontario Basketball Association, Ontario Football Alliance, Ontario Hockey Federation, Ontario Soccer Association and Athletics Ontario. CAO formally requested that these organizations distribute the link to their members in their own e-newsletters. One month after the initial communication, there were 270 completed surveys. The survey link was then made available to NTK members to distribute among their local sport organizations to increase the number of

TABLE 1

CAO members participating in a survey of spit tobacco use among youth athletes

\begin{tabular}{lcc} 
Sport & \multicolumn{2}{c}{ Coaches, $\mathbf{n}$} \\
& Population in CAO & $\begin{array}{c}\text { Sample size required for } \\
\text { subgroup analysis }\end{array}$ \\
\hline Baseball & 124 & 94 \\
Basketball & 969 & 276 \\
Football & 119 & 92 \\
Hockey & 244 & 150 \\
Soccer & 353 & 185 \\
Track and field & 113 & 88 \\
Total & 1922 & 321 \\
\hline
\end{tabular}

Abbreviation: CAO, Coaches Association of Ontario.

${ }^{a}$ Sample size estimate based on a margin of error of $5 \%$ and a confidence interval of $95 \%$.

responses. In broadening the population, statistical significance could no longer be computed. However, statistical significance would not have been attained based on the survey completion rate by CAO members alone due to the small sample size. It was also recognized that in changing the data collection strategy the population might carry an inherent geographical bias (e.g. different health regions may advocate more strongly than others); however, this also was offset by the need to increase the number of survey respondents.

A descriptive analysis including frequencies and cross-tabulations was completed using SurveyMonkey and Microsoft Excel 2000.

\section{Results}

There were 344 completed surveys on the SurveyMonkey web server. Of these, 83 were excluded because the respondents did not meet the eligibility criteria (coached a sport other than sports of interest, $\mathrm{n}=70$; coach reported only coaching athletes aged 8 years or less, $\mathrm{n}=9$, or 19 years or older, $\mathrm{n}=4$ ). The remaining 261 survey responses were analysed using descriptive statistics.

\section{Coaches}

Of those surveyed, the majority of coaches (72\%) were aged between 35 and 54 years, approximately $10 \%$ were aged 55 years plus and the remaining $20 \%$ were aged between 18 and 24 years. In addition, most were male $(76 \%)$.

\section{Geography}

Table 2 shows the geographic distribution of coaches. Geographic regions are based on Tobacco Control Area Networks (TCANs), created by the Ministry of Health Promotion and Sport under the Smoke-Free Ontario Strategy in order to coordinate regional initiatives, facilitate use of limited resources and tailor activities to suit specific contexts. ${ }^{23}$
TABLE 2

Geographic distribution of surveyed coaches, Ontario, Canada

\begin{tabular}{lc} 
TCAN region & Proportion, \% \\
\hline Central East & 29 \\
Central West & 34 \\
Eastern & 9 \\
North East & 5 \\
North West & 1 \\
South West & 13 \\
Toronto & 9 \\
Unknown & 2 \\
\hline
\end{tabular}

Abbreviation: TCAN, Tobacco Control Area Networks. 


\section{Sport}

The distribution of coaches by sport was uneven. Over a quarter $(29 \%)$ of respondents indicated they primarily coach baseball, and about the same proportion (28\%) reported that they mainly coach soccer. Basketball coaches $(17 \%)$ and hockey coaches (13\%) were also well represented. Football and track and field coaches each represented less than $10 \%$ of the sample respectively.

\section{Level of competition}

Most coaches reported coaching athletes at more than one level of competition, with the majority coaching at the competitive (provincial) level (59\%) and/ or recreational level $(43 \%)$.

\section{Frequency and duration of coaching}

Coaches at all levels of competition reported coaching two or more months out of the year. One-third (32\%) reported coaching their team for three to five years; however, $18 \%$ had been coaching for as little as less than one year, and $26 \%$ for as long as six or more years.

\section{Athletes}

Most coaches reported coaching athletes in many age categories. Overall, the largest proportion were aged 13 to 14 years. Just over half of the coaches (51\%) reported coaching all-male teams, though the sample also represented coaches of all-female teams (31\%) and of mixed teams $(26 \%)$.

\section{Self-perceived knowledge about ST}

Most coaches (68\%) reported knowing at least something about ST. More of the coaches aged 45 years plus had at least some knowledge of ST compared to coaches aged less than 45 years, $74 \%$ versus $58 \%$.

\section{Method of obtaining ST products}

Many coaches (29\%) believe athletes purchase ST products directly from vendors, for example, convenience stores, while the same proportion believe athletes are given ST products by friends, family and/ or acquaintances. Some coaches (15\%) believe athletes obtain ST through the "grey market" (e.g. Internet, street sales), $6 \%$ believe ST is taken from home without permission, $3 \%$ selected "Other," and $18 \%$ believe that athletes are not obtaining ST products.

\section{Perceived prevalence of spit tobacco use among athletes}

The majority of coaches $(78 \%)$ reported that none of the players on their teams used ST. Approximately 9\% reported that they have either witnessed their players using ST or they suspect that one or more players use ST, while $5 \%$ reported that they have witnessed players on other teams using ST. Ten per cent of respondents reported they do not know if any of their players use ST.

Only those coaching athletes aged 13 years plus reported ST use on their teams. However, a few coaches of athletes aged between 9 and 12 years indicated that they suspect ST use or they had witnessed ST use among athletes in the same age range on other teams.

All coaches who reported seeing one or more of their players using ST coached all-male teams. Only coaches of baseball, football and hockey reported witnessing athletes on their teams using ST.

\section{Knowledge about ST products and use}

The "True or False" section of the survey questioned coaches on their knowledge of ST products and use. The majority of coaches $(98 \%)$ indicated correctly that ST does not enhance athletic performance; that ST is not a safe alternative to smoking ( $98 \%)$; and that ST may cause mouth sores, gum recession and/or tooth loss (98\%). Of the respondents, $96 \%$ indicated correctly that ST use might contribute to high blood pressure, heart attacks and strokes, $52 \%$ agreed that "spit tobacco is a growing problem among youth practicing sports in Ontario these days," and $78 \%$ indicated correctly that it is illegal to give ST to youth younger than 19 years.

\section{Perceived role in addressing ST use}

Coaches were asked what they thought their role was with respect to addressing ST issues on their team. Over half indicated they would meet with and counsel their athletes (56\%) and/or inform the athlete's parents $(54 \%)$. Providing athletes with written information on ST was also a popular choice $(38 \%)$. Some also reported referring athletes to support services (e.g. health care providers, public health professionals, sport officials) and/ or informing the league organizer or other community agencies (e.g. leagues, schools) of any ST use (36\% and $25 \%$, respectively).

\section{Strategies used to address ST use}

Almost half of respondents (45\%) reported they have not done anything to influence their athletes regarding ST use and that they have not addressed ST use on their teams because they do not consider it to be an issue. Several, particularly coaches of female athletes, said they did not see a need to address ST use as their athletes were too young. Approximately one-quarter (23\%) indicated that they promote and/or enforce a tobacco-free sports policy.

\section{Useful ST information for coaches}

Coaches were asked to select the types of information that would be most helpful in advising youth about ST use. Almost half $(47 \%)$ indicated that information about the health effects of ST would be the most helpful (Table 3).

\section{Preferred means of receiving information on ST}

Many coaches indicated they would prefer to receive information about ST via the CAO website $(40 \%)$, in their coaching manuals, or in newsletters and pamphlets (Table 4). Since many of the survey respondents were CAO members and had likely accessed the survey through the CAO website, the preference for this format as a way to receive further information may not be true for the larger coaching population. A small proportion of respondents indicated they would prefer to attend workshops to 
TABLE 3

Types of information on spit tobacco that would be useful to coaches, by TCAN region, Ontario, Canada

\begin{tabular}{|c|c|c|c|c|c|c|c|c|}
\hline \multirow{2}{*}{$\begin{array}{l}\text { Type of information } \\
\text { deemed useful }\end{array}$} & \multicolumn{8}{|c|}{ Coaches by TCAN region, \% } \\
\hline & Central East & Central West & Eastern & North East & North West & South West & Toronto & All regions \\
\hline Different forms of ST & 29 & 22 & 25 & 13 & 0 & 29 & 34 & 26 \\
\hline $\begin{array}{l}\text { How to encourage athletes } \\
\text { to stay ST-free }\end{array}$ & 36 & 35 & 25 & 47 & 0 & 38 & 48 & 36 \\
\hline $\begin{array}{l}\text { How the tobacco industry } \\
\text { targets youth }\end{array}$ & 28 & 21 & 43 & 20 & 0 & 31 & 31 & 27 \\
\hline $\begin{array}{l}\text { I do not require information } \\
\text { at this time }\end{array}$ & 24 & 31 & 21 & 27 & 33 & 26 & 7 & 25 \\
\hline
\end{tabular}

Abbreviations: TCAN, Tobacco Control Area Networks; ST, spit tobacco.

TABLE 4

Coaches' preferred sources of information on spit tobacco, by TCAN region, Ontario, Canada

\begin{tabular}{|c|c|c|c|c|c|c|c|c|}
\hline \multirow[t]{2}{*}{ Preferred source of information } & \multicolumn{8}{|c|}{ Coaches by TCAN region, $\%$} \\
\hline & Central East & Central West & Eastern & North East & North West & South West & Toronto & All regions \\
\hline CAO website & 45 & 35 & 54 & 47 & 0 & 29 & 55 & 40 \\
\hline Workshops & 5 & 3 & 7 & 7 & 0 & 2 & 3 & 4 \\
\hline Newsletters and pamphlets & 31 & 37 & 46 & 7 & 0 & 38 & 34 & 34 \\
\hline
\end{tabular}

Abbreviations: CAO, Coaches Association of Ontario; TCAN, Tobacco Control Area Networks.

learn about the topic. In the "Other" category, most responses indicated email (from their sport club or the $\mathrm{CAO}$ ) as their preferred format to receive information.

\section{Discussion}

Our aim was to find out about coaches' knowledge of ST use among their athletes and their knowledge gaps with respect to ST use.

The majority of respondents reported they have not witnessed any ST use among athletes. Since this study was intended to direct health promotion activities rather than provide a prevalence estimate, the results of this investigation are not comparable with previous studies of self-reported ST use among athletes. ${ }^{13,17}$ Additional factors associated with differences in the study populations (e.g. age, geography and culture) may further distort comparisons with previous ST prevalence studies. However, our finding that only coaches of all-male teams observed ST use among their athletes is consistent with findings from other studies.
Many coaches report they have not done anything to address the issue of ST on their teams. However, rather than this being as a result of a lack of interest in their athletes, most coaches do not see the need to address ST use because they do not regard it as an issue on their teams. It is possible that this study demonstrates that coaches are not well aware of ST use among their athletes.

Coaches aged 45 years plus are more confident about their knowledge of ST products and use compared to younger coaches. In addition to their age, certain characteristics of coaches and athletes are important to consider since they relate to findings about coaches' knowledge and awareness of ST. For instance, results from this study are mostly applicable to all-male baseball, basketball, soccer and hockey teams.

While many coaches report that ST use is not a problem on their teams, almost three-quarters of the coaches said they would like more information on the topic. The results of the study provide insight into techniques that coaches currently use to address ST use among their players.

\section{Strengths and limitations}

The survey link was initially distributed only to coaches who were active members of CAO. As data collection progressed and it became apparent that sample size targets would not be met, the sampling strategy was amended. The survey link was distributed to a much broader population and distribution was not uniform across the province. As a result, there may be clustering among survey respondents, causing some responses to be more similar than would be expected if it were a random sample. Given the broad distribution and not knowing who received the survey, the results may not be generalizable to the larger population of coaches.

Out of 36 public health units in Ontario, 31 are members of NTK. As a result, and because of different strategies for promoting the survey to coaches by member health units, all areas in Ontario were not equally represented. Thus, the results of this survey may not be generalizable, particularly for underrepresented geographical areas. 
Since the sampling strategy changed during data collection, it is not possible to estimate a response rate. However, considering the response rate among CAO members was extremely low at the outset, electronic distribution of surveys might not be the most effective data collection strategy for this population. Another limitation associated with online surveys is the potential for bias among respondents. For example, a large proportion of respondents selected the CAO website as their preferred format to receive information; however, respondents who complete online surveys are likely to be more familiar with the virtual environment relative to the larger population of coaches.

Web-based survey programs have the inherent limitation of some degree of data insecurity. No identifying or personal information was collected in this study and all data was encrypted.

Despite the limitations of electronic survey distribution, this data collection method is efficient and inexpensive, making it well suited for a study involving a large sample of geographically dispersed respondents such as this one. Another advantage of online surveys is that there are clear start and end dates and results may be analyzed as soon as the data collection period ends.

Despite its limitations, this study is one of the first in Canada to shed light on an important and emerging issue.

\section{Conclusion}

Based on the survey results, coaches are fairly knowledgeable about the effects of ST products and ST use: almost $100 \%$ of coaches responded correctly to questions about the health effects of ST use, and about $80 \%$ of respondents correctly answered the question about legislation associated with ST and youth.

Most coaches are interested in receiving information about ST, particularly the health effects of ST use and how to prevent ST use among athletes. Multiple formats should be used to disseminate information to coaches. Both electronic (CAO website, emails from sport organizations, Health Unit website, etc.) and hard copy (included in coaching manuals, newsletters, pamphlets, etc.) formats were indicated as preferred methods to receive information about ST.

Our findings may be used to increase coaches' knowledge of ST use and ST effects in order to increase their ability to influence the tobacco use habits of their players. Coaches should learn more about ST products and their use, particularly as ST use is a growing issue in sport.

By better understanding coaches' perceptions of ST use and their information needs, public health professionals can develop initiatives that encourage coaches to take a more active role in ST prevention. These initiatives could include information and tools tailored to coaches' needs.

Since TCANs develop regional tobacco control plans and coordinate activities among member public health units, ${ }^{24}$ the results from this study may be used to direct TCAN health promotion and tobacco prevention activities as well as those of provincial groups, such as NTK.

Public health professionals in Ontario are currently focusing on tobacco-free sport and recreation (TFSR) policy as the next step in population-based tobacco control. Results from this study support the TFSR movement by adding new knowledge about coaches' awareness of the health effects of ST and their perception of ST use among Ontario athletes.

\section{Acknowledgements}

The authors would like to acknowledge the Not to Kids Coalition for supporting and funding this project. They would also like to acknowledge the individuals and organizations that contributed to the project:

- The Coaches Association of Ontario (CAO), specifically Susan Kitchen and Jessica Taggio, for their work in developing the survey and providing their expertise to the challenge of generating interest among coaches;

- The Haliburton, Kawartha, Pine Ridge District Health Unit for their support in conducting the analysis and writing this report;
- Theresa Chambers from the Simcoe Muskoka District Health Unit for her contribution to survey development;

- Coach respondents for volunteering their time and sharing their experience through the survey.

\section{References}

1. Youthography. Findings from qualitative studies into tobacco products and anti-tobacco creative development. Not to Kids Coalition; 2007. 116 p.

2. Ministry of Health and Long-Term Care. Ontario public health standards 2008 [Internet]. Toronto (ON): Ministry of Health and Long-Term Care; 2008 [cited 2010 Jul 28]. Available from: http:// www.health.gov.on.ca/english/providers /program/pubhealth/oph_standards/ophs /progstds/pdfs/ophs_2008.pdf

3. Sapundzhiev N, Werner JA. Nasal snuff: historical review and health related aspects. J Laryngol Otol. 2003;117:686-91.

4. Cooper J, Ellison JA, Walsh MM. Spit (smokeless)-tobacco use by baseball players entering the professional ranks. J Athl Train. 2003;38(2):126-32.

5. U.S. Department of Health \& Human Services (HHS). The health consequences of using smokeless tobacco: a report of the Advisory Committee to the Surgeon General [Internet]. Bethesda (MD): United States Public Health Service; 1986 Apr [cited 2011 Jan 28]. NIH Publication No. 86-2874. Available from: http://profiles.nlm.nih.gov /ps/access/NNBBFD.pdf

6. Tomar SL, Winn DM. Chewing tobacco use and dental caries among U.S. men. J Am Dent Assoc. 1999;130:1601-10.

7. Colilla SA. An epidemiologic review of smokeless tobacco health effects and harm reduction potential. Regul Toxicol Pharmacol. 2010;56:197-211.

8. Canadian Tobacco Use Monitoring Survey (CTUMS) 2008. Summary of annual results for 2008. Ottawa (ON): Health Canada; 2008 [modified 2010 Jan 22; cited 2011 Jan 28]. Available from: http://hc-sc.gc.ca /hc-ps/tobac-tabac/research-recherche /stat/_ctums-esutc_2008/ann_summary -sommaire-eng.php 
9. Propel Centre for Population Health Impact. Youth Smoking Survey 2008/2009: Smoking Profile for Ontario Youth [Internet]. Waterloo (ON): Propel Centre for Population Health Impact; 2009 [cited 2011 Aug 17]. Available from: http://www.yss.uwaterloo.ca/results /yss08_provincial_report_ON.pdf

10. Office of Applied Studies. The NSDUH report: smokeless tobacco use, initiation, and relationship to cigarette smoking: 2002 to 2007 [Internet]. Rockville (MD): Substance Abuse and Mental Health Services Administration; 2009 Feb 19 [cited 2009 Mar 5]. Available from: http://www.oas.samhsa.gov/2k9 /smokelessTobacco/smokelessTobacco.htm

11. Eaton DK, Kann L, Kinchen S, Sahnklin S, Ross J, Hawkins J, et al. Centers for Disease Control and Prevention. Morbidity and Mortality Weekly Report: Youth Risk Behavior Surveillance-United States, 2009 [Internet]. MMWR 2010 [cited 2011 Jan 28];59(SS-5):1-131. Available from: http:// www.cdc.gov/mmwr/pdf/ss/ss5905.pdf

12. Goebel LJ, Crespo RD, Abraham RT, Masho SW, Glover ED. Correlates of youth smokeless tobacco use. Nicotine Tob Res. 2000;2:319-25.

13. Gansky SA, Ellison JA, Rudy D, Bergert N, Letendre MA, Nelson L, et al. Cluster-randomized controlled trial of an athletic trainer-directed spit (smokeless) tobacco intervention for collegiate baseball athletes: results after 1 year. J Athl Train. 2005;40(2):76-87.

14. Castrucci BC, Gerlach KK, Kaurman NJ, Orleans CT. Tobacco use and cessation behavior among adolescents participating in organized sports. Am J Health Behav. 2004;28:63-71.

15. Melnick MJ, Miller E, Sabo DF, Farrell MP, Barnes GM. Tobacco use among high school athletes and nonathletes: results of the 1997 Youth Risk Behavior Survey. Adolescence. 2001;36:727-47.

16. Rigotti NA, Lee JE, Wechsler H. US college students' use of tobacco products: results of a national survey. JAMA. 2000;284(6):699-705.
17. DeHass DM. Substance use: NCAA study of substance use of college student athletes [Internet]. Indianapolis (IN): National Collegiate Athletic Association; 2006 [cited 2011 Jan 28]. Available from: http://www.ncaa.org/wps/wcm/connect / 007 d 81004 e0 dabfe 9 f 3 aff 1 ad 6 fc 8 b 25 /2006_substance_use_report.pdf?MOD $=$ AJPERES $\&$ CACHEID $=007 \mathrm{~d} 81004 \mathrm{e} 0 \mathrm{dabfe}$ 9f3aff1ad6fc8b25

18. Green GA, Uryasz FD, Petr TA, Bray CD. NCAA study of substance use and abuse habits of college student-athletes. Clin J Sport Med. 2001;11:51-6.

19. Bloom GA, Durand-Bush N, Schinke RJ, Salmela JH. The importance of mentoring in the development of coaches and athletes. Int J Sport Psychol. 1998;29:267-81.

20. Parrott R, Duggan A. Using coaches as role models of sun protection for youth: Georgia's "Got Youth Covered” Project. J Appl Commun Res. 1999;27:107-19.

21. Walsh MM, Ellison J, Hilton JF, Chesney M, Ernster VL. Spit (smokeless) tobacco use by high school baseball athletes in California. Tob Control. 2000;9(2);ii32-ii39.

22. Horn KA, Maniar SD, Dino GA, Gao X, Mechstroth RL. Coaches' attitudes toward smokeless tobacco and intentions to intervene with athletes. J Sch Health. 2000;70(3):89-94.

23. Ministry of Health Promotion. Comprehensive tobacco control: guidance document [Internet]. Toronto (ON): Ministry of Health Promotion (ON); 2010 [cited 2011 Aug 19]. Available from http:// www.mhp.gov.on.ca/en/healthy-communities / public-health / guidance-docs /ComprehensiveTobaccoControl.PDF 